Check for updates

Cite this: RSC Adv., 2017, 7, 19815

Received 21st November 2016 Accepted 20th March 2017

DOI: $10.1039 / c 6 r a 27096 d$

rsc.li/rsc-advances

\section{Development and characterization of promising Cremophor EL-stabilized o/w nanoemulsions containing short-chain alcohols as a cosurfactant}

\begin{abstract}
Liya Zeng, (D) Xin Xin and Yalin Zhang*
Nanoemulsions have attracted much attention due to their wide application in commercial industries such as pharmaceutics, food, beverages and skin care. Cremophor EL has been extensively used for emulsification, protection and solubilization of various lipophilic bioactive agents. However, their anaphylactoid hypersensitivity reactions are still challenging. Therefore, the aim of this study was to develop and characterize a promising, low-level Cremophor EL-stabilized o/w nanoemulsion using the spontaneous emulsification method for oral administration. From comparing the physicochemical properties (particle size and size distribution) of their emulsions, Cremophor has better emulsifying capacity than Tween and the droplet radius of emulsions can be reduced by increasing the surfactant concentration in current systems. The impact of various short-chain alcohols $(\mathrm{C}-\mathrm{OH}<5)$ on the formation and stability of nanoemulsions was also investigated to optimize the formulations. And there is a good correlation between nanoemulsion droplet size and the surfactant packing parameter $(p)$. The smallest droplet size with the narrowest size distribution was produced by the system containing ethylene glycol as a cosurfactant. However, preparation of nanoemulsions with ethylene glycol was a time-consuming process since it was difficult to emulsify the water-induced gel-structure. Surfactantto-oil mass ratio and surfactant-to-cosurfactant levels had to be optimized to produce fine droplets. Moreover, there was a linear relationship between the viscosity/log $P$ value of alcohols and droplet size. Ostwald ripening was the dominant breakdown process of nanoemulsions obtained in the ethyl oleate/ Cremophor EL-butanol $(3: 2) /$ water system. Ostwald ripening rate was increased with increasing surfactant concentration. Dilution $(100 \times)$ of this system considerably improved their stability against droplet growth. This study demonstrated that alcohol addition might be a useful tool for preparing Cremophor EL-stabilized nanoemulsions for pharmaceutical applications.
\end{abstract}

\section{Introduction}

Nano-emulsions are a class of emulsions covering the size range 20-500 nm. ${ }^{1,2}$ Due to their small droplet size, several differences in their physicochemical properties and functional behaviors are exhibited in contrast to conventional emulsions: transparent/translucent appearance, long-term stability and high bioavailability. Unlike microemulsions, which are thermodynamically stable, nano-emulsions are kinetically stable and can be produced at low surfactant levels, which is very important for industrial applications in pharmacy, ${ }^{3}$ food, ${ }^{4}$ cosmetics, ${ }^{5}$ agrochemistry ${ }^{6}$ and polymer synthesis. ${ }^{7}$ Because of the characteristic size of nano-emulsions, coalescence and Ostwald ripening are the main breakdown processes. ${ }^{7-9}$ Ostwald ripening arises from the difference in chemical potential

Key Laboratory of Plant Protection Resources \& Pest Management of the Ministry of Education, Entomological Museum, College of Plant Protection, Northwest A\&F University, Yangling 712100, Shaanxi, China. E-mail: yalinzh@nwsuaf.edu.cn; Fax: +8629 87092190; Tel: +862987092190 between small and large droplets, ${ }^{10}$ whereas coalescence results from droplet collisions. ${ }^{\mathbf{1 1}}$ Nano-emulsions can be fabricated using high-energy and low-energy methods. High-energy methods utilize mechanical devices to intermingle and disrupt the oil phase, ${ }^{12-16}$ while low-energy methods take advantage of the internal chemical energy of the components. ${ }^{17}$ Depending on the system and composition variables, similar droplet size can be achieved by both types of methods. ${ }^{\mathbf{4} 18-21}$ In the past decades, much attention has focused on low-energy emulsification methods due to simplicity of implementation, low cost and high energy efficiency.

Low-energy emulsification methods include the phase inversion temperature (PIT), phase inversion composition (PIC) and self-emulsification/spontaneous emulsification (SE) methods. Shinoda and Saito ${ }^{22,23}$ introduced the PIT method which relies on the changes in the surfactant curvature induced by temperature. However, in most cases the PIT method was only applicable to ethyoxylated/polyoxyethylene-type nonionic surfactants which are sensitive to changes in temperature. In the SE method, emulsion is formed when the oil-surfactant 
mixture is injected into the aqueous phase without any phase transition, whereas in the PIC method, emulsion is formed when water is added to an oil-surfactant mixture involving the inversion of the surfactant film. ${ }^{17,24}$ In the current study, we focused on the potential of spontaneous emulsification (SE) to fabricated Cremophor EL-stabilized nanoemulsions that are suitable for oral administration. Previous studies and reviews on nanoemulsions formed by spontaneous emulsification or self-emulsification methods can be found elsewhere. ${ }^{25-28}$ Nevertheless, the requirement of a high synthetic surfactant concentration is the main disadvantage for practical applications. It has been reported that inclusion of another amphiphile, such as cosurfactant/cosolvent, can alter the emulsification efficiency of surfactant, reduce the oil-water interfacial tension and consequently be a potential method for further reducing the surfactant concentration. ${ }^{29}$ Moreover, a cosurfactant/cosolvent with high hydrophilicity can diffuse from the oil phase to the aqueous phase, and the fine oil droplets are obtained only when the oil phase is completely converted to a liquid crystalline phase or microemulsion. ${ }^{\mathbf{1 7 2 5}}$ Previous studies have indicated that the order of addition, stirring speed, temperature, surfactant-to-oil ratio (SOR) and dilution procedures have an appreciable impact on the properties (e.g. droplet size and size distribution) and stability of nano-emulsions. ${ }^{30,31}$ However, most of these previous model systems (e.g., water/ $\mathrm{C}_{12} \mathrm{E}_{6} /$ medium- (long-) chain alcohol/ hydrocarbon, water/SDS/co-surfactant/dodecane and water/Brij 30/hexadecane) are not suitable for industrial applications.

The heterogeneous non-ionic surfactant Cremophor EL (polyoxyl 35 castor oil) is a pale yellow oily liquid $\left(25^{\circ} \mathrm{C}\right.$ ) with an approximate critical micelle concentration (CMC) that lies at $0.02 \%$, the hydrophilic-lipophilic balance (HLB) that lies between 12 and 14, and it is produced by reaction of castor oil with ethylene oxide at a molar ratio of $1: 35 .^{32}$ Much attention has been focused on the application of Cremophor EL in pharmaceutical industries, which can be attributed to its ability to solubilize, protect and encapsulate the lipophilic bioactive compounds. ${ }^{33-36}$ A good example would be the pharmaceutical formulation of the anticancer agent paclitaxel, which containing 50\% Cremophor EL, 50\% dehydrated ethanol and $30 \mathrm{mg}$ paclitaxel. ${ }^{33}$ In addition, a number of selfnanoemulsifying drug delivery systems (SNEDDS) containing Cremophor EL that enhanced the permeability and bioavailability of drugs has been reported. ${ }^{33,37}$ Cremophor RH 40 (polyoxyl hydrogenated 40 castor oil) is another frequently used castor oil derivative. In consideration of the unsaturation and lower degree of ethoxylation, we speculated that the particle size and size distribution of emulsions emulsified by Cremophor EL tend to be smaller and narrower than those emulsified by Cremophor RH 40. Unfortunately, Cremophors are usually associated with severe anaphylactoid hypersensitivity reactions, hyperlipidaemia, abnormal lipoprotein patterns, aggregation of erythrocytes and peripheral neuropathy. ${ }^{33}$ To reduce these side effects, some methods have been employed, such as replacing the Cremophor with Solutol HS 15 (ref. 38) and adding the cosurfactant/cosolvent. ${ }^{39}$ To our best knowledge, there are few reports describing the formation, properties and stability of nano-emulsions stabilized by Cremophor. Additionally, most of these reported nanoemulsions contained high levels of cosolvents/co-surfactants or were produced by high-energy methods. ${ }^{29,36}$ Consequently, to further investigate a Cremophor EL-stabilized pharmaceutical formulation with low levels of surfactant/co-surfactant prepared by spontaneous emulsification method is important.

In this study, our main objective was to investigate the possibility of producing pharmaceutical o/w nanoemulsions by the spontaneous emulsification method at low Cremophor-EL levels. Ethyl oleate was selected as the oil in this study to avoid reacting chemically with surface-active species, such as free fatty or polar lipids. Moreover, ethyl oleate is generally considered to be pharmaceutical acceptable and widely used in drug delivery systems. It has been determined that the addition of cosurfactant had an appreciable effect on the formation and stability of emulsion-based systems. ${ }^{27,40,41}$ Since little research has focused on the role of short-chain alcohols in the available spontaneous emulsification systems, ethanol, ethylene glycol, 1-propanol, propylene glycol, isopropanol, and 1-butanol were selected as co-surfactants (Table 1). In order to deepen

Table 1 Physical properties of alcohols used to prepare nanoemulsions by the spontaneous emulsification method and the relationship between $Z$-average droplet diameters/size distribution of nanoemulsion with Cremophor EL $(1,2)$ and Cremophor RH $40\left(1^{\prime}, 2^{\prime}\right)$. The physicochemical properties were measured at ambient temperature $\left(25^{\circ} \mathrm{C}\right)$. The correlation coefficients $\left(r^{2}\right)$ were calculated from plots of droplet size $($ correlation 1) and PDI (correlation 2) versus the physicochemical property of interest

\begin{tabular}{|c|c|c|c|c|c|}
\hline Co-surfactants & $\begin{array}{l}\text { Molecular weight } \\
\left(\mathrm{g} \mathrm{mol}^{-1}\right)\end{array}$ & HLB & $\begin{array}{l}\log P \\
\text { (octanol/water) }\end{array}$ & $\begin{array}{l}\text { Viscosity } \\
(\mathrm{mPa} \mathrm{s})\end{array}$ & $\begin{array}{l}\text { Emulsification } \\
\text { time (sec) }\end{array}$ \\
\hline Ethanol & 46.07 & 4.2 & -0.30 & 1.08 & $<60$ \\
\hline Ethylene glycol & 62.07 & 9.85 & -1.2 & 16.1 & $<60$ \\
\hline 1-Propanol & 60.10 & 7.48 & 0.25 & 1.94 & $<60$ \\
\hline Propylene glycol & 76.1 & 9.38 & -0.79 & 2.04 & $<60$ \\
\hline Isopropanol & 60.10 & 7.4 & 0.05 & 2.1 & $<60$ \\
\hline 1-Butanol & 74.12 & 7.0 & 0.88 & 2.57 & $<60$ \\
\hline Correlation $_{1}\left(r^{2}\right)$ & -0.20 & -0.13 & 0.25 & 0.65 & - \\
\hline Correlation $_{2}\left(r^{2}\right)$ & -0.18 & -0.07 & 0.33 & 0.11 & - \\
\hline Correlation $_{1^{\prime}}\left(r^{2}\right)$ & -0.25 & 0.25 & 0.80 & 0.15 & - \\
\hline Correlation $_{2^{\prime}}\left(r^{2}\right)$ & -0.24 & -0.24 & -0.25 & -0.09 & \\
\hline
\end{tabular}


understanding of the mechanisms of spontaneous emulsification at constant temperature, the influence of surfactant type and concentration, various short-chain alcohols and their concentration on the droplet size and size distribution were also described. Since SNEDDS are often highly diluted in the gastrointestinal tract (GIT), the robustness of dilution and longterm stability were also of concern. Moreover, recognizing the primary instability mechanism of emulsions containing cosurfactant is necessary to find the most efficient strategy to improve their stability. In particular, this study provides important information about the influence of Cremophor-EL and short-chain alcohols on the formation, properties and stability of SNEDDS for pharmaceutical applications.

\section{Experimental}

\subsection{Materials}

Ethyl oleate (purity $\geq 98 \%$ ), ethanol (purity $\geq 99.8 \%$ ), ethylene glycol (purity $\geq 99 \%$ ), 1-propanol (purity $\geq 99.5 \%$ ), propylene glycol (purity $\geq 99 \%$ ), isopropanol (purity $\geq 99.5 \%$ ), and 1butanol (purity $\geq 99.7 \%$ ) were obtained from Aladdin $\AA$ (Shanghai, China). Cremophor RH 40 and Cremophor EL were obtained from the BASF Corporation (Germany). All other chemicals and solvents were of analytical grade and used as supplied without any further purification.

Deionized water was generated from a Milli-Q gradient system of Millipore (Synergy, Millipore SAS, Molsheim, France).

\subsection{Nano-emulsions preparation}

Nano-emulsions were prepared by the spontaneous emulsification (SE) method described in a previous study ${ }^{\mathbf{4 2}}$ with slight modifications. Briefly, SE was performed by adding the organic phase into an aqueous phase by mildly stirring at $25{ }^{\circ} \mathrm{C}$. The organic phase was composed of ethyl oleate, Cremophor EL and co-surfactant. Each sample was further stirred for 15 min (500 $\mathrm{rpm})$. Unless otherwise stated, the final water content was kept constant at $96 \mathrm{wt} \%$.

\subsection{Phase diagram determination}

Ternary phase diagrams were plotted to evaluate the dynamic phase behavior of all types of formulations. Aqueous titration method was used for the construction of pseudo ternary phase diagrams. Surfactants and co-surfactants were mixed at different weight ratios (Smix $=$ surfactant : co-surfactant $=$ $4: 1,3: 1,2: 1,1: 1,1: 0,1: 2,1: 3)$. Nine different weight ratios of Smix to oil (SOR $=1: 9,2: 8,3: 7,4: 6,5: 5,6: 4$,
$7: 3,8: 2,9: 1)$ were employed to precisely delineate the boundaries of phases formed in the phase diagram. The required amounts of oil and surfactant were weighted accurately, gently heated to $50{ }^{\circ} \mathrm{C}$ and mixed. All components were mixed at ambient temperature $\left(25^{\circ} \mathrm{C}\right)$. The nano-emulsion phase was identified as the region in the phase diagram where it was transparent/translucent in appearance and an easily flowable formulation was obtained. Optically anisotropic liquid crystalline phases were identified by polarized light microscopy (OptipHot-2, Nikon, Japan). Attempts were also made to distinguish between micelles, o/w nano-emulsions (Om), W/O nano-emulsions (Wm), and bicontinuous (D) or liquid crystalline phases (LC).

\subsection{Thermodynamic stability study}

In order to assess the physical stability of the selected formulations the effect of the following stresses were analyzed:

Centrifugation test. ${ }^{43-45}$ The formulations were centrifuged at $5000 \mathrm{rpm}$ for $30 \mathrm{~min}$ and observed for phase separation, creaming or cracking.

Heating-cooling cycle. The formulations after the centrifugation test were stored at refrigerated temperature $\left(4^{\circ} \mathrm{C}\right)$ and $40{ }^{\circ} \mathrm{C}$ (six cycles each) for $48 \mathrm{~h}$ at each temperature.

Freeze-thaw cycle consisted of three freeze-thaw cycles for “ $-21{ }^{\circ} \mathrm{C}, 48 \mathrm{~h}$ and $+25^{\circ} \mathrm{C}, 48 \mathrm{~h}$.

\subsection{Dispersibility study}

The effect of dispersity of oral nanoemulsions was assessed using a standard USP XXII dissolution apparatus $2.1 \mathrm{~mL}$ of each formulation was added to $500 \mathrm{~mL}$ of water and $0.1 \mathrm{~N} \mathrm{HCl}$ respectively at $37 \pm 0.5^{\circ} \mathrm{C}$. A standard stainless steel dissolution paddle rotating at $50 \mathrm{rpm}$ provided gentle agitation. The in vitro performance of the formulations was visually assessed using the grading system shown in Table 2 and the results of these dispersibility studies are shown in Table 3.

\subsection{Droplet size and polydispersity}

Dynamic light scattering (DLS) (Nano ZS, Malvern Instrument Ltd, Worcestershire, UK) at a fixed scattering angle of $173^{\circ}$ and with the laser wavelength of $633 \mathrm{~nm}$ was used for measuring the $Z$-average droplet size, particle size distribution (PSD) and polydispersity index (PDI). Measurements were conducted at $25^{\circ} \mathrm{C}$ and each measurement was made with three readings per sample. Additionally, measurements were carried out for all samples after equilibrating for $2 \mathrm{~h}$.

Table 2 Observation table of dispersibility study

\begin{tabular}{|c|c|c|}
\hline & Observation & Grad \\
\hline A & Rapidly forming (within $1 \mathrm{~min}$ ) nanoemulsion, having a clear or bluish appearance & A \\
\hline B & Rapidly forming, slightly less clear emulsion, having a bluish white appearance & B \\
\hline $\mathrm{C}$ & Fine milky emulsion that formed within 2 minutes & $\mathrm{C}$ \\
\hline $\mathrm{D}$ & Dull, grayish white emulsion, having slightly oily appearance, that is slow to emulsify (longer than 2 min) & $\mathrm{D}$ \\
\hline $\mathrm{E}$ & Formulation, exhibiting either poor or minimal emulsification, with large oil globules present on the surface & $\mathrm{E}$ \\
\hline
\end{tabular}


Table 3 Dispersibility study of different samples selected from the ethyl oleate/Cremophor EL/butanol/water system (Smix $=2: 1$ )

Observations based on the dispersibility studies

\begin{tabular}{llll} 
SOR & Distilled water & $0.1 \mathrm{~N} \mathrm{HCl}$ & Inference \\
\hline $16 / 1$ & A & A & Passed \\
$14 / 3$ & A & A & Passed \\
$12 / 5$ & A & A & Passed \\
$10 / 7$ & B & B & Passed \\
$8 / 9$ & B & B & Passed \\
$6 / 11$ & C & C & Failed
\end{tabular}

\subsection{Stability}

The long-term stability of the nano-emulsions was assessed by measuring the droplet size of nano-emulsions as a function of time by DLS $\left(25^{\circ} \mathrm{C}\right)$. The samples were prepared by diluting the original SNEDDS by 100 times to negate the effects of multiple scattering.

\subsection{Data analysis}

All statistical tests were repeated three times and expressed as the mean $\pm \mathrm{SE}$.

\section{Results and discussion}

\subsection{Cremophor and Tween}

Nonionic or zwitterionic surfactants are often considered for SNEDDS based on their high degree of compatibility with other components, good physicochemical stability, low toxicity and being less affected by $\mathrm{pH}$ and ionic strength changes. ${ }^{37}$ A number of previous studies have investigated the influence of nonionic surfactants on nanoemulsion delivery systems produced by the SE method. ${ }^{29}$ These studies proposed that the properties of a surfactant, such as the HLB (hydrophile-lipophile balance) number, viscosity and the affinity for the oil phase, have an appreciable effect on the emulsification process and nanodroplets formation. In this section, we selected two types of nonionic surfactants (Cremophor and Tween), since these surfactants are usually utilized for the development of pharmaceutical emulsions. Cremophor has been associated with severe anaphylactoid hypersensitivity reactions, hyperlipidaemia and peripheral neuropathy etc., ${ }^{33}$ though Cremophor has better emulsifying capacity than Tween in most case. And Cremophor EL had been replaced with Tween 80 to decrease the neuronal damages. ${ }^{46}$ Cremophor RH 40 and Cremophor EL are the popular castor oil derivatives (Table 4), while Cremophor EL has a slightly lower degree of ethoxylation and is not hydrogenated. Tween 80 is derived from polyethoxylated sorbitan and oleic acid whereas Tween 20 is formed by the ethoxylation of sorbitan before the addition of lauric acid. They are primarily different in the nature of the hydrophobic tail group. To examine the influence of surfactant type on the size of droplets, a series of emulsions with fixed oil (ethyl oleate, $50 \mathrm{wt} \%$ ) were prepared using different surfactant types (Cremophor and Tween, $50 \mathrm{wt} \%$ ) but under a similar stirring speed (500 rpm) and thermal environment (25 $\left.{ }^{\circ} \mathrm{C}\right)$. The weight ratio of oil-to-surfactant and the oil content were decided on the basis of previous investigations for spontaneously emulsifying systems. ${ }^{37,47,48}$

The type of nonionic surfactant has a considerable impact on the droplet size and size distribution (PDI) (Table 4). In current study, the smallest droplets with the narrowest size distribution were obtained with the Cremophor RH $40(r \approx 66.79 \mathrm{~nm}, \mathrm{PDI} \approx$ 0.15) as surfactant, whereas the largest droplets with the broadest distribution were formed in the system prepared using Tween 20 ( $r \approx 179.2 \mathrm{~nm}$, PDI $\approx 0.29$ ). In addition, as can be seen from Table 4 , the Cremophors have a better emulsifying capacity than the Tweens. Nevertheless, Musa et al. ${ }^{\mathbf{4 9}}$ and Ammar et $a .^{50}$ found that Tween 80 as surfactant provided a smaller droplet as well as PDI than Cremophor EL. We did not find a correlation between the droplet dimension and HLB value of surfactant (e.g., Tween $20(r \approx 179.2 \mathrm{~nm}$, PDI $\approx 0.29)$ and Tween $80(r \approx 122.73 \mathrm{~nm}, \mathrm{PDI} \approx 0.16)$ has similar HLB numbers but exhibited different droplet diameters). This phenomenon is similar to the production of vitamin D nanoemulsion-based delivery systems using the spontaneous emulsification method..$^{51}$ Moreover, no correlation was found between droplet diameter and the molecular weight of a nonionic surfactant. This result is in opposite to the previous works which suggested that the small-molecule surfactants are more effective at producing the small droplets than polymer surfactant. ${ }^{52,53}$ Predictably, there are other factors that need to be considered in our study.

Previous researches have suggested that molecular characteristics of surfactant can appreciably influence the formation of self-emulsification nanoemulsions. ${ }^{51,52,54}$ The molecular geometry of a surfactant can be described by its surfactant packing parameter $(p)$, which is defined as $v_{\mathrm{o}} / a_{\mathrm{e}} l_{\mathrm{o}}$, where $v_{\mathrm{o}}$ and

Table 4 Effect of nonionic surfactant type with different chemical structure and HLB on particle diameter and polydispersity index of emulsions. 4 wt \% oil-in-water emulsions were prepared at room temperature $\left(25^{\circ} \mathrm{C}\right)$ using 50 wt $\%$ ethyl oleate and 50 wt $\%$ surfactant and a stirring speed of $500 \mathrm{rpm}$

\begin{tabular}{|c|c|c|c|c|c|}
\hline Surfactants & Chemical structure & $\begin{array}{l}\text { Molecular weight } \\
\left(\mathrm{g} \mathrm{mol}^{-1}\right)\end{array}$ & HLB number & Droplet size $(r, \mathrm{~nm})$ & PDI \\
\hline Cremophor EL & Polyoxyl 35 castor oil & $\approx 1630$ & $12-14$ & $73.47 \pm 1.55$ & $0.18 \pm 0.04$ \\
\hline Cremophor RH 40 & Polyoxyl 40 hydrogenated castor oil & $\approx 2500$ & $14-16$ & $66.79 \pm 0.40$ & $0.15 \pm 0.06$ \\
\hline Tween 20 & Polyoxyethylen-20 sorbitan monolaurate & 1228 & 16.7 & $179.20 \pm 7.31$ & $0.29 \pm 0.02$ \\
\hline Tween 80 & Polyoxyethylen-20 sorbitan monooleate & 1310 & 15 & $122.73 \pm 3.09$ & $0.16 \pm 0.03$ \\
\hline
\end{tabular}


$l_{\mathrm{o}}$ are the volume and the length of the surfactant tail and $a_{\mathrm{e}}$ is the equilibrium area per molecule at the aggregates surface.$^{55} \mathrm{In}$ other words, if we know the molecular packing parameters of a surfactant, the shape and size of the equilibrium aggregate can be readily identified ( $0 \leq v_{\mathrm{o}} / a_{\mathrm{e}} l_{\mathrm{o}} \leq 1 / 3$ for a sphere, $1 / 3 \leq v_{\mathrm{o}}$ ) $a_{\mathrm{e}} l_{\mathrm{o}} \leq 1 / 2$ for a cylinder, and $1 / 2 \leq v_{\mathrm{o}} / a_{\mathrm{e}} l_{\mathrm{o}} \leq 1$ for bilayers). Additionally, differences in surfactant packing parameters influence the interfacial characteristics such as the curvature of the monolayer, which is generated by surfactant molecules spontaneously associate with each other in water and has a curvature allowing the most efficient packing of the surfactant molecules. ${ }^{4}$ The change in spontaneous curvature of a surfactant during the emulsification process has been recognized to be a key factor for the nanoemulsion formation. Tween 20 and Tween 80 have similar polar head-groups, but Tween 20 has a monolaurate tail $\left(\mathrm{C}_{12: 0}\right)$ and Tween 80 has a monooleate tail $\left(\mathrm{C}_{18: 1}\right)$. Thus, Tween 80 would be expected to have bigger packing parameter than Tween 20. On the contrary, Cremophor EL and Cremophor RH 40 have similar non-polar tail-groups, but Cremophor RH $40(n=40)$ with high degree of polymerization than Cremophor EL $(n=35)$. Hence, Cremophor RH 40 may have a smaller packing parameter than Cremophor EL. We therefore postulate that there is an intermediate packing parameter which could be expected to impact the formation of fine oil droplets. In addition, the packing parameter of Cremophor may be closer to this intermediate value than Tween.

\subsection{Influence of alcohol type on nanoemulsion formation}

Large amounts of surfactant may cause gastrointestinal irritation when administered orally; it is therefore important to determine the surfactant concentration properly or add cosurfactant/cosolvent into the formulations to reduce the surfactant concentration. A single surfactant alone may not be able to achieve the extremely low oil/water interfacial tension and fluid interfacial film for producing the nanoemulsion, hence addition of a cosurfactant is appealing. The water/ Cremophor/cosurfactant/ethyl oleate systems, using a shortchain length alcohol (Table 1) as cosurfactant, were investigated to determine the influence of cosurfactant type on the properties (i.e., droplet size and size distribution) of nanoemulsions. As the mass ratio of surfactant to cosurfactant is constant (Smix $=3: 2$ ), the ability of short-chain alcohols to improve the emulsification of surfactant is clearly distinguished. All of the studied cosurfactants are pharmaceutically acceptable ingredients.

A series of $4 \mathrm{wt} \%$ oil-in-water nanoemulsions were prepared in the absence and presence of short-chain alcohols using the spontaneous emulsification method. The addition of alcohols had a significant impact on the final droplet size and size distribution (Fig. 1), which is in line with previous studies. ${ }^{\mathbf{4 1 5 4}}$ In the absence of alcohol, the droplet sizes of emulsions with Cremophor EL and Cremophor RH 40 were $73.47 \mathrm{~nm}$ and $66.79 \mathrm{~nm}$, respectively. On the other hand, in the presence of alcohols, there was a decrease in the droplet size in the nanoemulsions produced using Cremophor EL or Cremophor RH 40 (Fig. 1). Furthermore, the emulsification time was obviously

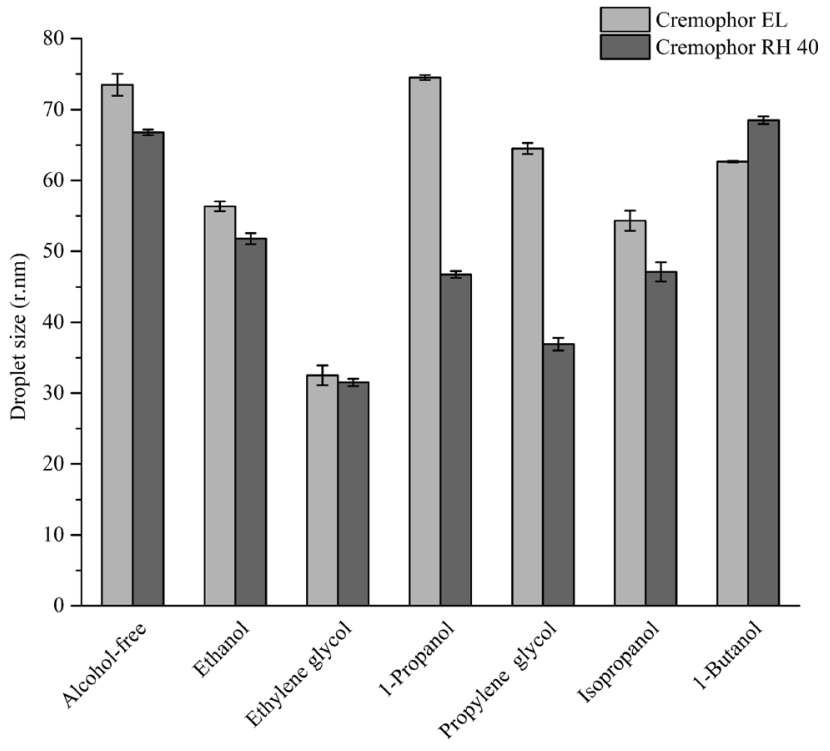

Fig. 1 Effect of short-chain alcohol addition on the droplet size (Zaverage) of $4 \%$ wt nano-emulsions $\left(25^{\circ} \mathrm{C}\right)$. Ethyl oleate (50 wt\%) was used as the oil phase, Cremophor EL and Cremophor RH 40 as surfactant, and the mass ratio of surfactant-to-cosurfactant Smix = $3: 2$.

reduced with the presence of alcohols. These differences in the appearances of produced nanoemulsions may be attributed to the fact that the alcohols have a noticeable impact on the interfacial characteristics, such as spontaneous curvature, interfacial tension, interfacial fluidity, polarity and thickness. In addition, alcohols may also modify the physicochemical properties of aqueous solutions such as density, refractive index, viscosity and polarity. In order to deepen the mechanisms for nanoemulsions formation with the presence of alcohols, droplet size distributions were measured by dynamic light scattering (Fig. 2). Interestingly, the measurement of PDI indicated that most of these nanoemulsions showed a monomodal PSD. This result indicates that the alcohols may not have a major impact on droplet rupture and/or the droplet growth have occurred immediately after spontaneous emulsification. ${ }^{\mathbf{5 6}}$ Previous work has shown that the addition of cosurfactants can influence the formation of nanoemulsions prepared by high pressure homogenization: smaller droplets were obtained by addition of short-chain alcohols due to their ability to change the viscosity of the aqueous phase and influence the mass transports kinetics of surfactant and oil molecule. ${ }^{\mathbf{4 1}}$

Furthermore, the emulsification efficiency of surfactant is improved by the addition of alcohols (emulsification time, Table 1). This performance may be affected by the molecular structure and chain-length of alcohols. As can be seen from Fig. 1 and 2, there are obvious variations in the droplet size and size distribution of produced nanoemulsions, which depend on the type of alcohols. The smallest droplets were produced when using ethylene glycol as cosurfactant no matter what type of surfactant was used, and then using propylene glycol and isopropanol mixed with Cremophor RH 40. The relatively large droplets $(>60 \mathrm{~nm}$ ) could be formed using 1-propanol mixed with 


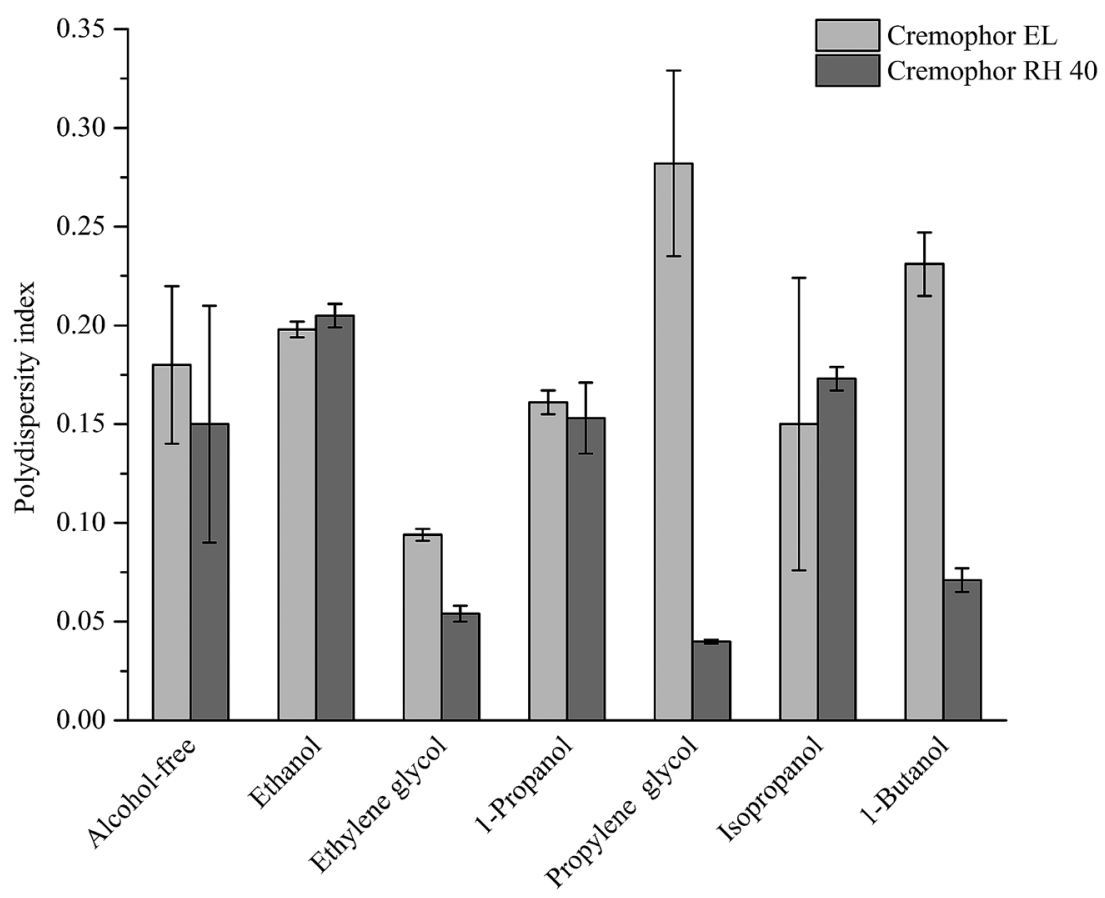

Fig. 2 Effect of short-chain alcohol addition on the size distribution (PDI) of $4 \%$ wt nano-emulsions $\left(25^{\circ} \mathrm{C}\right)$. Ethyl oleate $(50$ wt $\%)$ was used as the oil phase, Cremophor EL and Cremophor RH 40 as surfactant, and the mass ratio of surfactant-to-cosurfactant Smix $=3: 2$.

Cremophor EL. We hypothesized that there would be a correlation between the physicochemical properties of the alcohols and nano-droplets formation, such as molecular weight, HLB, $\log P$ or viscosity. Zeeb et al. reported that the $\log P$ value may influence the partitioning of alcohols into the dispersed phase and this phenomenon associated with the increase in hydrophobicity with the increasing molecular weight of alcohols. ${ }^{41}$ Additionally, it was also reported that an oil with higher viscosity could produce smaller droplets by the SE method. ${ }^{42}$ Therefore, we correlated the droplet diameter with the physicochemical properties of the varied alcohols: molecular weight, HLB, $\log P$ and viscosity. We found that there was no correlation $\left(r^{2}<0.3\right)$ between molecular weight/HLB and droplet size (Table 1). Interestingly, there was a good correlation between alcohol viscosity and droplet size $\left(r^{2}=0.65\right.$, Cremophor EL as surfactant) and alcohol $\log P$ value and droplet size $\left(r^{2}=0.80\right.$, Cremophor RH 40 as surfactant). It is likely that the surfactant molecular structure cause this difference in correlation, because the addition of alcohols could improve the penetration of the oil phase in the nonpolar section of a surfactant, thereby further decreasing the interfacial tension. This result suggests that it is possible to produce commercial nanoemulsions by selecting the proper cosurfactant.

\subsection{Construction of phase diagrams}

Phase diagrams were constructed to evaluate all types of formulations that were composed of oil, surfactant (cosurfactant) and water. In addition, the relationship between the components of the nanoemulsion and their phase behaviors can be provided by constructing a phase diagram. The phase diagrams were examined for the following two systems. Ethyl oleate/Cremophor EL-short-chain alcohols $(3: 2) /$ water and ethyl oleate/Cremophor RH 40-short-chain alcohols $(3: 2) /$ water are shown in Fig. 3a and Fig. 3b, respectively. The area occupied by triangle dots indicates the area in which nanoemulsions of desired size and stability were obtained. It is noteworthy that the anisotropic phase was obtained when the surfactant concentration was less than $20 \%$. As expected the type and the chain-length of the alcohols had notable influence on the amount of oils solubilized within the nanoemulsions. From Fig. 3a, it can be seen that ethyl oleate/Cremophor EL/butanol system exhibits the largest nanoemulsification region. Within the short-chain alcohols of co-surfactants, butanol, the longest chain-length alcohol, could be incorporated into the interfacial film and then solubilized the oils at approximately $60 \mathrm{w} / \mathrm{w} \%$. Similarly to Cremophor RH 40, the largest nanoemulsion region was presented in the ethyl oleate/Cremophor RH 40/butanol system, where similar oils were solubilized (Fig. 3b). These results indicate that butanol has a greater self-emulsification efficiency than other short-chain alcohols. These results were as expected and follow the trend recorded in the previous literature. ${ }^{41}$ Nevertheless, the nanoemulsion regions of Cremophor EL systems were similar to that of Cremophor RH 40. This may be attributed to their similar HLB values and molecular structures, which support them as highly suitable for development of self-nanoemulsifying emulsions.

Interestingly, there is no remarkable difference between the nanoemulsion area of the ethylene glycol system and the butanol system. Ethylene glycol, having a shorter hydrocarbon chain compared to butanol, has higher water-solubility; therefore, it has a higher tendency to diffuse from the oil phase into 

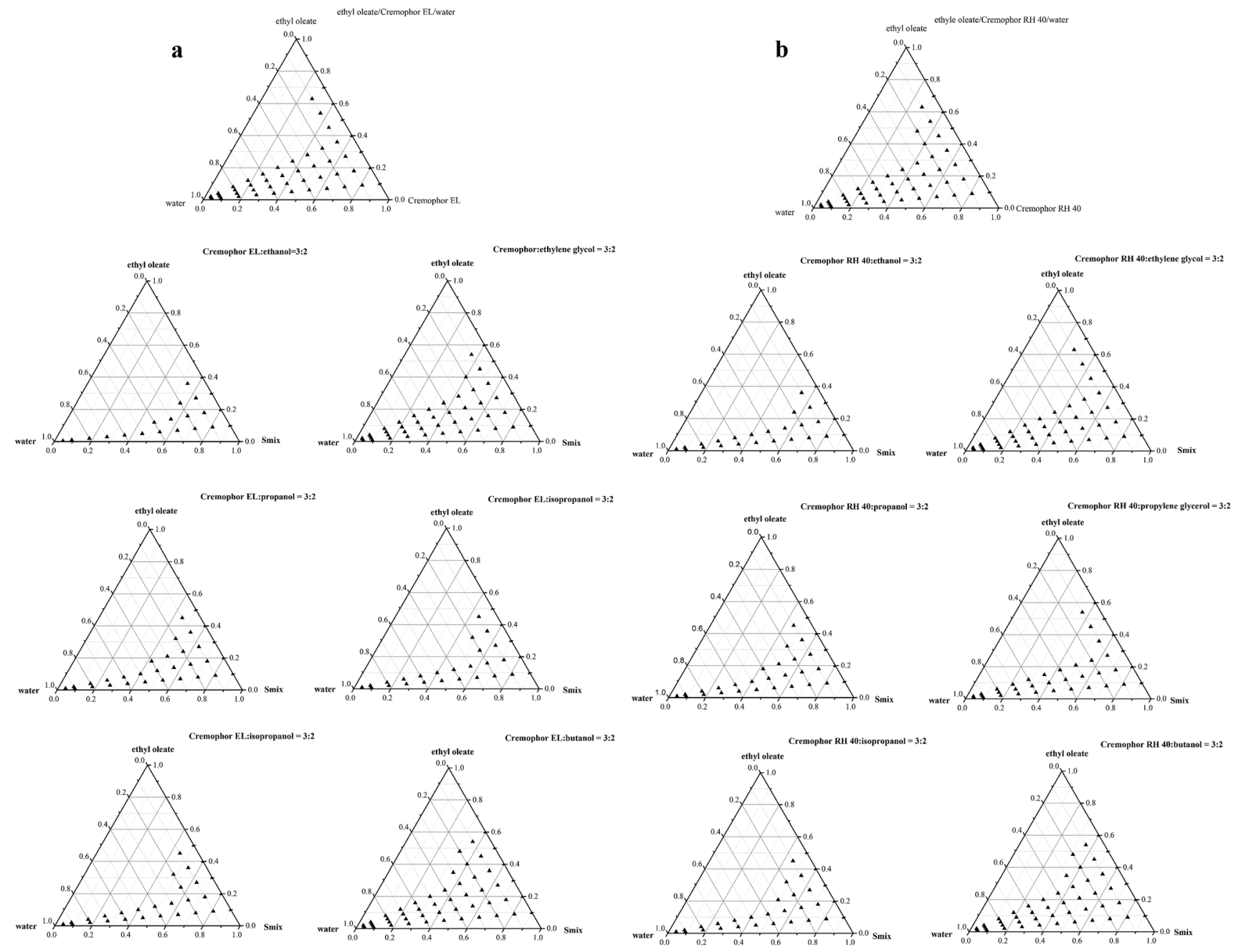

Fig. 3 (a) Pseudo-ternary phase diagrams of ethyl oleate with Cremophor EL as surfactants to get the nanoemulsion area (denoted by solid triangles) with different co-surfactants at a fixed Smix ratio (3:2). (b) Pseudo-ternary phase diagrams of ethyl oleate with $\mathrm{Cremophor} \mathrm{RH} 40$ as surfactants to get the nanoemulsion area (denoted by solid triangles) with different co-surfactants at a fixed Smix ratio (3:2).

the aqueous phase during the dilution process. Previous theoretical and experimental studies have demonstrated that the rheological properties of the oil and aqueous phases can influence the droplet disruption and stability. ${ }^{9,53}$ It is clear that ethylene glycol has a higher viscosity (16.1 mPa s, Table 1$)$ than other studied alcohols. Based on the Taylor equation, $r \sim \gamma /$ $(\eta c \dot{\gamma}$ ), (where $r$ is the mean droplet diameter, $\gamma$ is the interfacial tension, $\eta_{\mathrm{c}}$ is the continuous phase viscosity and $\dot{\gamma}$ is the shear rate) the efficiency of droplet breakup increase may be due to a raising of the continuous phase via rapid diffusion of ethylene glycol into the aqueous phase. In addition, the addition of ethylene glycol expanded the region of the gel-like phase from the phase diagrams compared to the alcohol-free systems. The high viscosity of the gel-like phase could prevent the droplet coalescence and uniform distribution of droplets.

The influence of the surfactant-to-cosurfactant mass ratio (Smix) on the formation of a nanoemulsion was also investigated. In view of Fig. 3, due to a larger nanoemulsion region and greater capacity for incorporating oils, the ethyl oleate/ Cremophor EL/butanol/water system was studied (Fig. 4).
When surfactant was used alone, a large gel-like phase was observed, while an appreciable nanoemulsion region was obtained towards the water-rich apex. The maximum concentration of oil that could be solubilized was $70 \% \mathrm{w} / \mathrm{w}$. This appearance shows that Cremophor EL can be used alone in the absence of cosurfactant, but the emulsification time is too long. When cosurfactant was incorporated along with the surfactant in equal ratios (Smix $=1: 1$ ), the nanoemulsion region was slightly diminished but the maximum amount of oil that could be emulsified was $60 \% \mathrm{w} / \mathrm{w}$. In addition, the formation of a nanoemulsion became gradually easier and more flowable. This may be attributed to the combination of surfactant and cosurfactant which enhance the flexibility of the surfactant layer, decrease the interfacial tension and increase the entropy of the system. With the increase of cosurfactant ( $\operatorname{Smix}=1: 2$ ), the total nanoemulsion area was further reduced making it just $20 \% \mathrm{w} / \mathrm{w}$ oil solubilized. Conversely, when the surfactant concentration was increased as in the Smix to $2: 1$, the nanoemulsion region was similar to when it was $1: 1$. When we increased the 

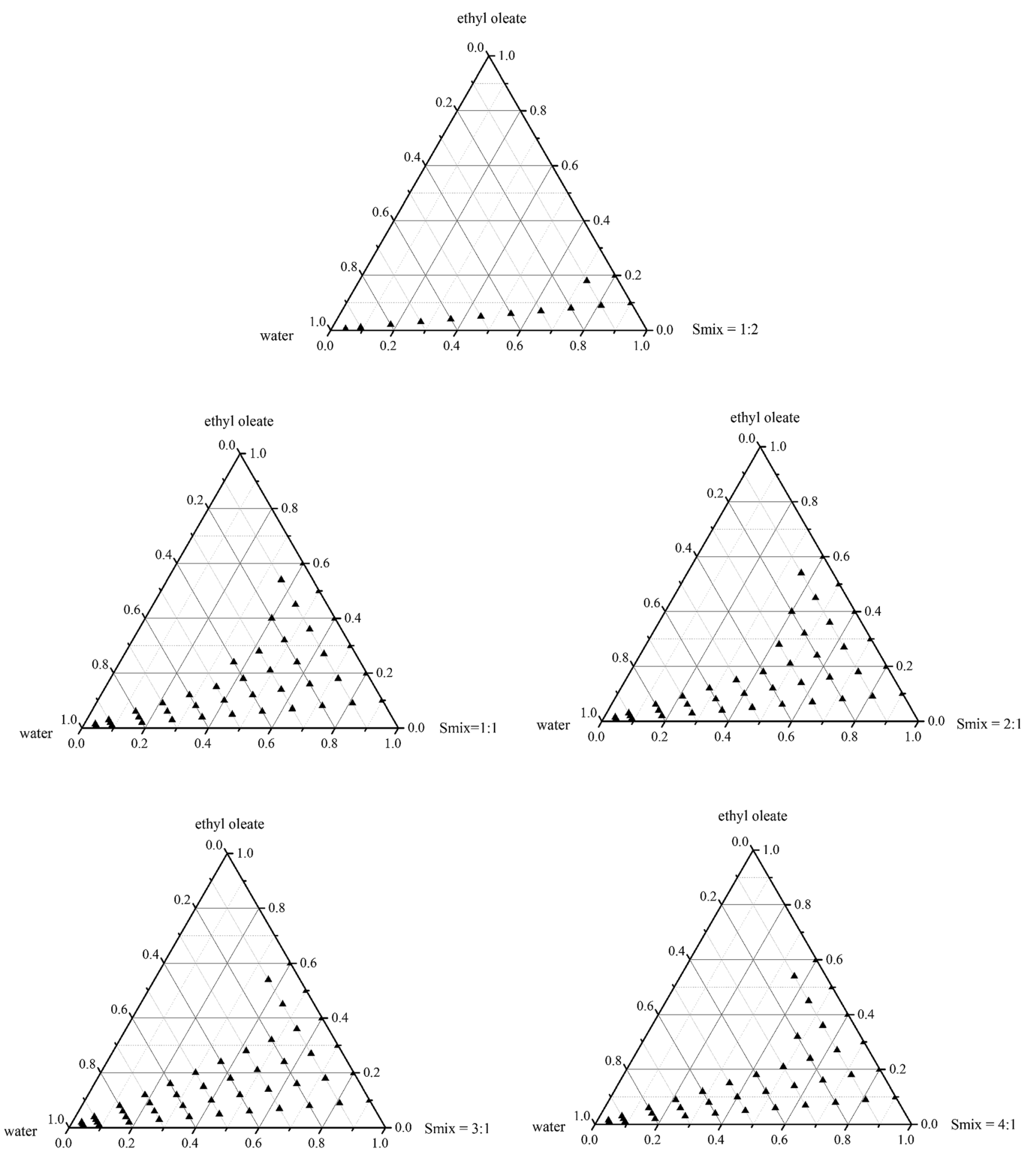

Fig. 4 Pseudo-ternary phase diagrams of ethyl oleate with Cremophor EL as surfactant to get nanoemulsion area (denoted by solid triangles) with butanol as cosurfactant at different Smix ratios $(1: 2,1: 1,2: 1,3: 1,4: 1)$.

surfactant concentration to $3: 1$ (Smix), the nanoemulsion area increased and the gel-like phase started appearing in the phase diagram. However, there was no change in the maximum amount of oil that could be emulsified when the Smix ranged from $1: 1$ to $3: 1$. Further increasing the Smix to $4: 1$, the spontaneity of the self-emulsification process slowed with the increasing gel phase. An appreciable decrease in the nanoemulsion region was observed but the maximum solubilized oil concentration still remained the same as that of $1: 1$. Similar observations were reported in previous literatures where the combination of surfactant and cosurfactant increased emulsification efficiency., 


\subsection{Influence of surfactant concentration}

The effect of Cremophor EL concentration on droplet size was investigated by preparing a series of emulsions with fixed oil (ethyl oleate) and Smix (Cremophor EL : butanol = $2: 1$ ) but with different surfactant-to-oil ratios (SOR). The mean particle droplet size and polydispersity index (PDI) followed similar trends with increasing SOR (Fig. 5). The mean droplet size increased with the surfactant concentration decreased, which is in agreement with former studies. ${ }^{52,58}$ This phenomenon is attributed to several reasons: the reduction in interfacial tension with an increasing surfactant concentration and the surfactant diffusion into the aqueous phase facilitating the formation of fine droplets. ${ }^{54}$ The PDI was lowest at the highest surfactant concentration $(S O R=16 / 1)$. Our results indicate that a nanoemulsion containing relatively small oil droplets $(d<60 \mathrm{~nm})$ can be formed at a lower SOR (8/9). This value is much higher than the value needed to fabricate the novel Cremophor EL-based nanoemulsions using high energy methods such as ultrasound cavitation, ${ }^{59}$ but is similar to that reported for the formation of ramipril nanoemulsions by spontaneous emulsification. ${ }^{57}$ Small droplets $(r<100 \mathrm{~nm})$ were also produced when the surfactant level further decreased (SOR < 8/9), but a noticeable phase separation was observed on standing for about $2 \mathrm{~h}$. An interesting phenomenon was that the droplet diameter increased as the surfactant concentration rose. We speculate that the highest surfactant concentration (SOR $=16 / 1$ ) exceeded the certain surfactant level where the formation of a highly viscous liquid crystalline phase could make spontaneous breakup of the oilwater interface more difficult. ${ }^{52,54}$ Consequently, there is an optimum surfactant concentration required to form uniformly distributed droplets, which must be determined for each surfactant, cosurfactant, oil and water combination.

\subsection{Thermodynamic stability studies}

Nanoemulsions can be expected to approximate thermodynamically stable systems and form at a particular concentration

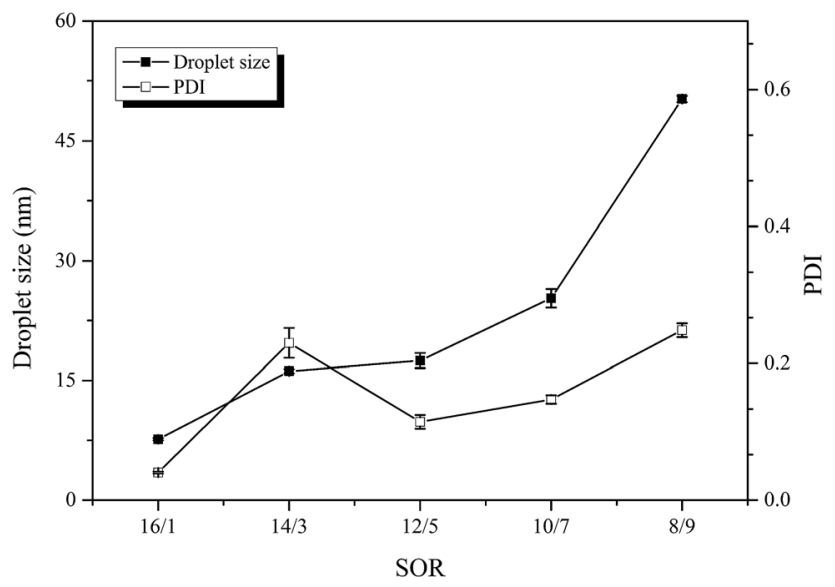

Fig. 5 Effect of Cremophor EL concentration (surfactant-to-oil mass ratio, SOR) on mean droplet diameter and polydispersity index (PDI) of emulsions produced by spontaneous emulsification. $4 \mathrm{wt} \%$ emulsions were prepared using fixed ethyl oleate and Smix (Cremophor $\mathrm{EL}:$ butanol $)=2: 1$ at a stirring speed of $500 \mathrm{rpm}\left(25^{\circ} \mathrm{C}\right)$. of oil, surfactant and water. In addition, the physicochemical properties of nonionic surfactant can be appreciably affected as the temperature varies, associated with hydration and dehydration of the nonionic surfactant head-group. To avoid selection of metastable systems, thermodynamic stability studies such as the heating cooling cycle, centrifugation and freeze thaw cycle were performed. It was observed that formulations (SOR $\geq 8 / 9$ ) prepared from ethyl oleate/Cremophor EL-butanol $(2: 1)$ /water system passed the thermodynamic stress tests and were then selected for the dispersibility test.

\subsection{Dispersibility studies}

Because the objective of this study was to develop a promising oral nanoemulsion stabilized by Cremophor EL, dispersibility studies in distilled water and $0.1 \mathrm{~N} \mathrm{HCl}$ were essential. Normally, the samples that passed the dispersibility test in distilled water as well as in $0.1 \mathrm{~N} \mathrm{HCl}$ in grade A and grade B (Table 4) were considered to pass the dispersibility test. In ethyl oleate/Cremophor EL-butanol (2:1)/water systems, almost all samples passed the dispersibility test. It should be noted that the samples failed dispersibility when the surfactant concentration was below 8/9. This means that there might be creaming or phase separation of nanoemulsions (SOR $<8 / 9$ ), which led to drug precipitation because of its poor water solubility.

\subsection{Stability}

Commercially, it is important that emulsion-based delivery systems remain stable during storage, transport, and utilization. Due to their small droplet size, nanoemulsions are resistant to gravitation separation/creaming and flocculation. Usually, the major destabilization mechanisms of nanoemulsions are Ostwald ripening and/or coalescence. And the growth rate and breakdown mechanisms of a nanoemulsion are dependent on its composition, microstructure and environmental conditions. We therefore examined the influence of surfactant concentration and storage time on the stability of Cremophor EL stabilized-nanoemulsions containing butanol as cosurfactant at ambient temperature.

The changes in droplet size as a function of storage time for nano-emulsions with different surfactant concentrations are shown in Fig. 6. Notably, when the surfactant concentration is below 8/9 (SOR), a distinct cream layer was observed on top of emulsions (data not shown here). We can see from Fig. 6 that all emulsions exhibited good stability by $100 \mathrm{~h}$. In all samples, the droplet size grows very slowly and the growth rate decreases with increasing surfactant concentration. For example, $r$ increased from $16.79 \mathrm{~nm}$ initially to $57.34 \mathrm{~nm}$ for $12 / 5$ SOR and from $50.29 \mathrm{~nm}$ to $69.50 \mathrm{~nm}$ for $8 / 9$ SOR. The width of the PSD was not appreciably affected by storage time with the PDI values changing slightly throughout $100 \mathrm{~h}$ storage: PDI increased from 0.136 to 0.187 for $12 / 5$ SOR and decreased from 0.242 to 0.158 for $8 / 9 \mathrm{SOR}$. This indicates the ethyl oleate/Cremophor EL/ butanol/water system is relatively stable to droplet growth under these conditions. 


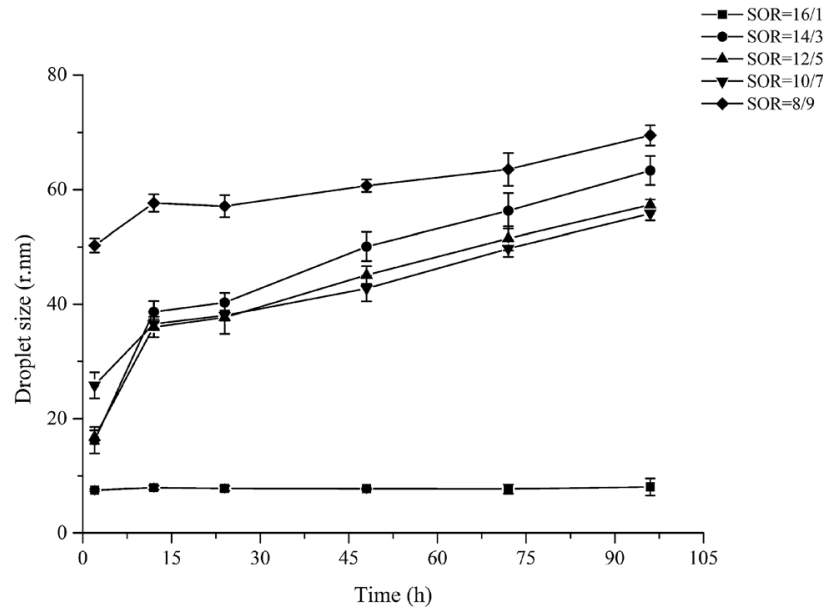

Fig. 6 Variations in the $Z$-average droplet size with different nanoemulsions (ethyl oleate/Cremophor EL-butanol (3 : 2)/water).

Ostwald ripening arises from the differences in chemical potential of droplet at different sizes. The difference in chemical potential of dispersed droplets is described by the Kelvin equation:

$$
C(r)=C_{\infty} \exp \left[\frac{2 \gamma V_{\mathrm{m}}}{r R T}\right]
$$

where $C(r)$ is the solubility of the emulsified oil, $C_{\infty}$ is the bulk solubility of the oils in the continuous phase, $r$ is the drop radius, $\gamma$ is the interfacial tension, $V_{\mathrm{m}}$ is the oils molar volume, $R$ is gas content and $T$ is the absolute temperature. In an emulsion with droplets of different sizes, larger droplets grow at the expense of smaller ones due to a different chemical potential through the continuous phase. Lifshitz, Slyozow, and Wagner proposed a theory, referred to as the LSW theory, for predicting the rate of Ostwald ripening:

$$
\omega=\frac{\mathrm{d} r^{3}}{\mathrm{~d} t}=\frac{8}{9}\left[\frac{C(\infty) \gamma V_{\mathrm{m}} D}{\rho R T}\right]
$$

where $\omega$ is the rate of Ostwald ripening, $D$ is the diffusion coefficient of the dispersed phase in the continuous and $\rho$ is the density of the dispersed phase.

In order to assess whether Ostwald ripening was the predominant destabilization mechanism of nano-emulsions obtained in the ethyl oleate/Cremophor EL/butanol/water systems, the cubic droplet size was plotted as a function of time (Fig. 7). According to eqn (2), the linear relationship between the $r^{3}$ and time was obtained for systems containing surfactant concentration below 16/1, indicating that the predominant destabilization is Oswald ripening. From the slope of each straight line, the Ostwald ripening rate can be determined (Table 5). As given in Table 5, the Ostwald ripening rate increases with increasing surfactant concentration from SOR $10 / 7$ to $14 / 3$. This phenomenon is always explained by the presence of free surfactant micelles in water, which transferred oil molecules between droplets. ${ }^{54}$ Our results are contradictory to that obtained by Liu et al. ${ }^{60}$ and Tong et al. ${ }^{61}$ which suggest

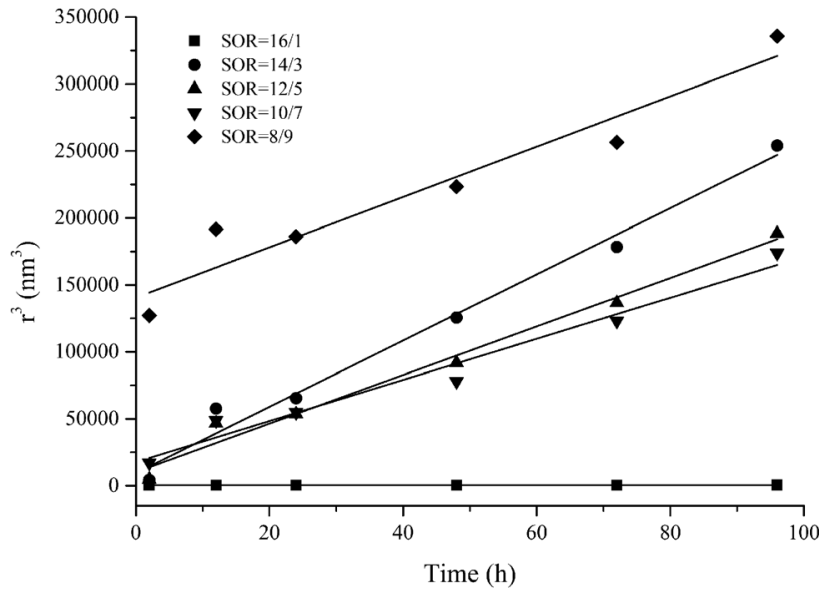

Fig. $7 \quad r^{3}$ vs. time for nanoemulsions prepared using the system ethyl oleate/Cremophor EL-butanol (3:2)/water at various surfactant concentration (SOR).

that the Ostwald ripening rate declines gradually with the increase of surfactant concentration.

Nevertheless, Wang and Davis have discussed that the time scale for Brownian-motion induced coalescence is proportional to the cube of the average drop radius. ${ }^{62}$ If coalescence is the driving forces for instability, the change of droplet radius with time may follow the following equation:

$$
\frac{1}{r^{2}}=\frac{1}{r_{0}^{2}}-\frac{8 \pi}{3 \omega t}
$$

where $r$ is the average droplet radius after time $t, r_{0}$ is the value at $t=0$, and $\omega$ is the frequency of rupture per unit of surface of the film. Table 5 shows that there was a good correlation between storage time and droplet size $(\mathrm{SOR}=8 / 9)$. This implies that both coalescence and Ostwald ripening are the predominant instability mechanisms at an SOR of $8 / 9$. Such instability may be attributed to the decreasing of surfactant concentration and a broadening of the PSD. In order to confirm this result, the changes in PSD over time are shown in Fig. 8; PSD broadening can be attributed to coalescence whereas PSD sharpening is a result of Ostwald ripening. ${ }^{8}$ In the first $24 \mathrm{~h}$, a gradual shift of bimodal size distribution to monomodal distribution towards higher droplet size through the expense of smaller droplets was observed. And then the particle distribution broadened with time, associated with the rise in the droplet size. Therefore, the variations in the particle size distribution indicate that not Ostwald ripening but coalescence is the main droplet breakdown processes.

The influence of dilution $(100 \times$ with water) on the storage stability of nanoemulsions was also examined (Fig. 9). This is very important for the practical application of emulsion-based delivery systems. After dilution the surfactant and cosurfactant concentrations decreased significantly. However, the droplet size of nanoemulsions after $100 \mathrm{~h}$ storage was still below $60 \mathrm{~nm}$. In addition, compared to undiluted emulsions, the growth rate of nanoemulsion was obviously retarded by dilution. The storage stability of nanoemulsions produced by the 
Table 5 Experimentally-determined Ostwald ripening rate and coalescence rate for a series of Cremophor EL stabilized o/w nanoemulsions $\left(25^{\circ} \mathrm{C}\right)$

\begin{tabular}{llll}
\hline $\begin{array}{l}\text { Surfactant concentration } \\
(\mathrm{SOR})\end{array}$ & $\begin{array}{l}\text { Ostwald ripening } \\
\text { rate }\left(\omega, \mathrm{nm}^{3} \mathrm{~s}^{-1}\right)\end{array}$ & $\begin{array}{l}\text { Correlation } \\
\left(r^{2}\right)\end{array}$ & $\begin{array}{l}\text { Coalescence } \\
\text { rate }\left(\mathrm{nm}^{-2} \mathrm{~s}^{-1}\right)\end{array}$ \\
\hline $16 / 1$ & 0.5038 & 0.0831 & $9.86548 \times 10^{-6}$ \\
$14 / 3$ & 2474.6397 & 0.9819 & $1.45221 \times 10^{-5}$ \\
$12 / 5$ & 1812.6377 & 0.9795 & $1.28723 \times 10^{-5}$ \\
$10 / 7$ & 1534.2723 & 0.9646 & $3.31022 \times 10^{-6}$ \\
$8 / 9$ & 1878.2075 & 0.9135 & $3.54204 \times 10^{-7}$
\end{tabular}

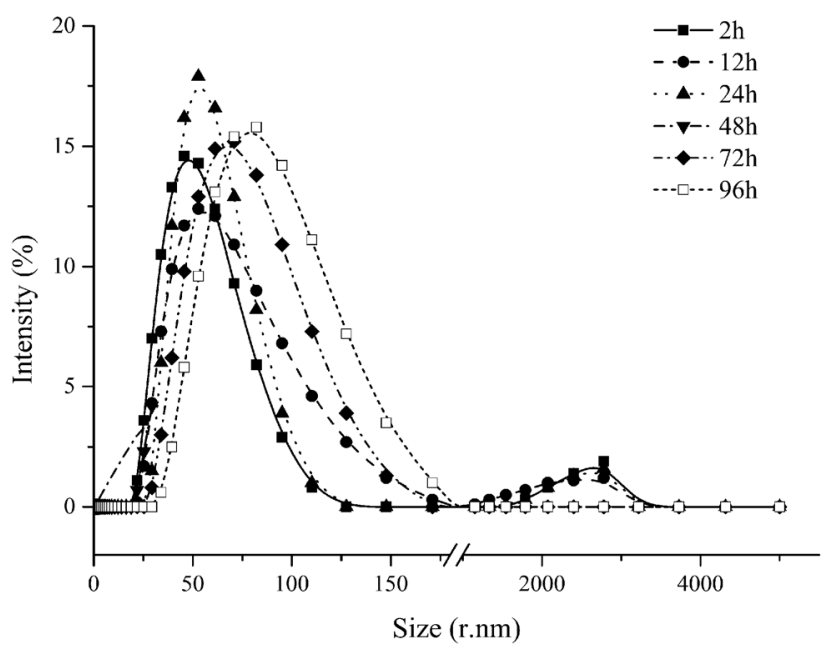

Fig. 8 Time evolution of PSD of nanoemulsion at SOR $8 / 9\left(25^{\circ} \mathrm{C}\right)$.

low-energy method can be improved by diluting them and the influence of dilution with water or cosurfactant solution have been examined in previous studies. ${ }^{27,54}$ We speculated that the dilution dissociates droplets of nanoemulsion and decreases the interactions between the hydrophilic surfactant head group.

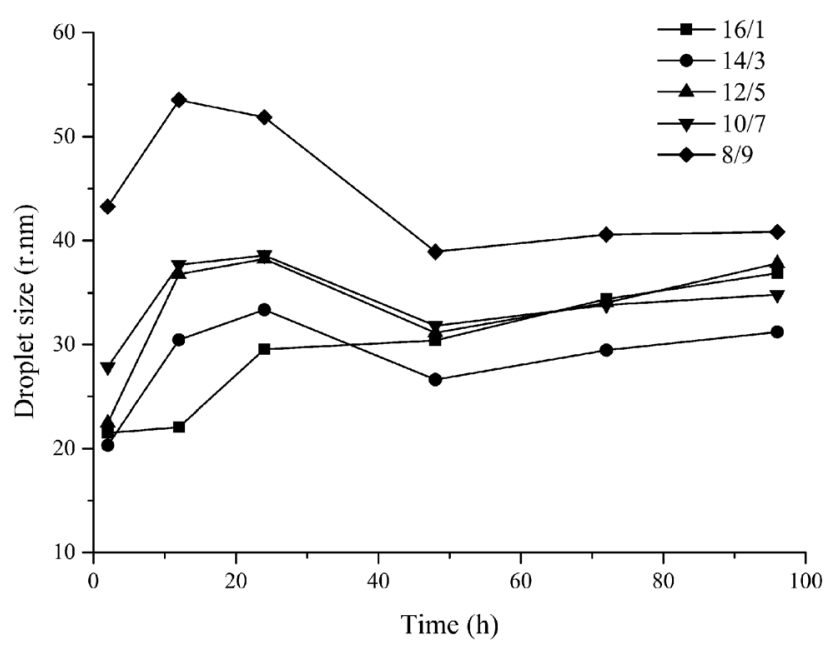

Fig. 9 Variation in the Z-average droplet size with different diluted nanoemulsions (ethyl oleate/Cremophor EL-butanol (3:2)/water, $100 \times)$.

\section{Conclusion}

In this study, we examined the effect of short-chain alcohols on the formation of low Cremophor EL concentration-stabilized nanoemulsions prepared by spontaneous emulsification. Alcohol addition had an appreciable impact on the formation, properties and stability of nanoemulsions due to their ability to alter surfactant solubility characteristics and optimum curvature. In addition, alcohol addition can reduce the total amount of surfactant required to produce fine nanoemulsions by spontaneous emulsification. But we also found that systems with relatively high cosurfactant content were extremely unstable. The initial droplet size and size distribution of nanoemulsions formed using the spontaneous emulsification were associated with the viscosity and hydrophilicity $(\log P)$ of alcohols. The smallest droplets with narrowest distribution were obtained when ethylene glycol was added to Cremophor EL stabilized nanoemulsion. However, the emulsification of this system was time-consuming due to the high viscosity of ethylene glycol, which may be a potential sustained-release delivery system. The main demulsification mechanism for the nanoemulsions stabilized by Cremophor EL was Ostwald ripening, whereas Ostwald ripening and coalescence both contribute to the destabilization of the nanoemulsions when the surfactant concentration is below 10/7 (SOR). Dilution $(100 \times)$ of nanoemulsions with water improved their stability to droplet growth, which was attributed to the reduction of surfactant interactions. This study provided a promising approach to producing fine nanoemulsions with low levels of surfactant, which is considerably important for commercial delivery systems.

\section{Acknowledgements}

The authors are grateful to the School of Life Sciences (Northwestern Polytechnical University, China) for supporting the use of the Malvern Zetasizer photo correlation spectroscopy (Nano ZS, Malvern Instrument, UK) and also sincerely appreciate John Richard Schrock (Emporia State University, USA) for revising the manuscript. This research is supported by the Special Fund for the Public Interest (Agriculture) (200903052) by The Ministry of Science and Technology, The Ministry of Agriculture of China, the '13115' Sci-Tech Innovation Project of Shaanxi Province (2007ZDKG-14, 2009ZDKG-06) and the Fundamental Research Funds for the Central Universities (2452015113). 


\section{References}

1 M. Sivakumar, S. Y. Tang and K. W. Tan, Ultrason. Sonochem., 2014, 21, 2069-2083.

2 C. Solans and I. Solé, Curr. Opin. Colloid Interface Sci., 2012, 17, 246-254.

3 H. Choudhury, B. Gorain, S. Karmakar, E. Biswas, G. Dey, R. Barik, M. Mandal and T. K. Pal, Int. J. Pharm., 2014, 460, 131-143.

4 D. J. McClements, Soft Matter, 2011, 7, 2297-2316.

5 O. Sonneville-Aubrun, J. T. Simonnet and F. L'Alloret, Adv. Colloid Interface Sci., 2004, 108-109, 145-149.

6 L. Chaw Jiang, M. Basri, D. Omar, M. B. Abdul Rahman, A. B. Salleh, R. N. Z. Raja Abdul Rahman and A. Selamat, Pestic. Biochem. Physiol., 2012, 102, 19-29.

7 C. Solans, P. Izquierdo, J. Nolla, N. Azemar and M. Garciacelma, Curr. Opin. Colloid Interface Sci., 2005, 10, 102-110.

8 E. Nazarzadeh, T. Anthonypillai and S. Sajjadi, J. Colloid Interface Sci., 2013, 397, 154-162.

9 T. J. Wooster, M. Golding and P. Sanguansri, Langmuir, 2008, 24, 12758-12765.

10 E. R. Garrett, J. Pharm. Sci., 1962, 51, 811-833.

11 G. Urbina-Villalba, A. Forgiarini, K. Rahn and A. Lozsan, Phys. Chem. Chem. Phys., 2009, 11, 11184-11195.

12 I. Alzorqi, M. R. Ketabchi, S. Sudheer and S. Manickam, Ultrason. Sonochem., 2016, 31, 71-84.

13 K. W. Tan, S. Y. Tang, R. Thomas, N. Vasanthakumari and S. Manickam, Pure Appl. Chem., 2015, 88, 43-60.

14 S. Parthasarathy, T. Siah Ying and S. Manickam, Ind. Eng. Chem. Res., 2013, 52, 11829-11837.

15 S. Y. Tang and M. Sivakumar, AIChE J., 2013, 59, 155-167.

16 T. K. Wei and S. Manickam, Asia-Pac. J. Chem. Eng., 2012, 7, S125-S133.

17 C. Solans and I. Solé, Curr. Opin. Colloid Interface Sci., 2012, 17, 246-254.

18 S. Y. Tang, P. Shridharan and M. Sivakumar, Ultrason. Sonochem., 2013, 20, 485-497.

19 S. Y. Tang, M. Sivakumar and B. Nashiru, Colloids Surf., B, 2013, 102, 653-658.

20 S. Y. Tang, M. Sivakumar, A. M.-H. Ng and P. Shridharan, Int. J. Pharm., 2012, 430, 299-306.

21 S. Y. Tang and M. Sivakumar, Asia-Pac. J. Chem. Eng., 2012, 7, S145-S156.

22 K. Shinoda and H. Saito, J. Colloid Interface Sci., 1968, 26, 7074.

23 K. Shinoda and H. Saito, J. Colloid Interface Sci., 1969, 30, 258-263.

24 I. Sole, C. M. Pey, A. Maestro, C. Gonzalez, M. Porras, C. Solans and J. M. Gutierrez, J. Colloid Interface Sci., 2010, 344, 417-423.

25 M.-J. Rang and C. A. Miller, J. Colloid Interface Sci., 1999, 209, 179-192.

26 C. A. Miller, Colloids Surf., 1988, 29, 89-102.

27 A. H. Saberi, Y. Fang and D. J. McClements, J. Colloid Interface Sci., 2013, 411, 105-113.
28 J. C. López-Montilla, P. E. Herrera-Morales, S. Pandey and D. O. Shah, J. Dispersion Sci. Technol., 2002, 23, 219-268.

29 A. A. Date, N. Desai, R. Dixit and M. Nagarsenker, Nanomedicine, 2010, 5, 1595-1616.

30 I. Sole, C. Solans, A. Maestro, C. Gonzalez and J. M. Gutierrez, J. Colloid Interface Sci., 2012, 376, 133-139.

31 J. Komaiko and D. J. McClements, J. Colloid Interface Sci., 2014, 425, 59-66.

32 H. Hoffman, Pharm. Ztg., 1984, 129, 1730-1733.

33 H. Gelderblom, J. Verweij, K. Nooter and A. Sparreboom, Eur. J. Cancer, 2001, 37, 1590-1598.

34 S. Shafiq, F. Shakeel, S. Talegaonkar, F. J. Ahmad, R. K. Khar and M. Ali, Eur. J. Pharm. Biopharm., 2007, 66, 227-243.

35 W. Li, S. Yi, Z. Wang, S. Chen, S. Xin, J. Xie and C. Zhao, Int. J. Pharm., 2011, 420, 161-171.

36 S. Y. Tang, S. Manickam, T. K. Wei and B. Nashiru, Ultrason. Sonochem., 2012, 19, 330-345.

37 E. B. Basalious, N. Shawky and S. M. Badr-Eldin, Int. J. Pharm., 2010, 391, 203-211.

38 B. Gorain, H. Choudhury, A. Kundu, L. Sarkar, S. Karmakar, P. Jaisankar and T. K. Pal, Colloids Surf., B, 2014, 115, 286294.

39 J.-L. Feng, Z.-W. Wang, J. Zhang, Z.-N. Wang and F. Liu, Colloids Surf., A, 2009, 339, 1-6.

40 J. Ahmad, S. R. Mir, K. Kohli and S. Amin, Colloids Surf., A, 2014, 453, 68-77.

41 B. Zeeb, E. Herz, D. J. McClements and J. Weiss, J. Colloid Interface Sci., 2014, 433, 196-203.

42 K. Bouchemal, S. Briancon, E. Perrier and H. Fessi, Int. J. Pharm., 2004, 280, 241-251.

43 V. Bali, M. Ali and J. Ali, Colloids Surf., B, 2010, 76, 410-420.

44 H. Choudhury, B. Gorain, S. Karmakar, E. Biswas, G. Dey,

R. Barik, M. Mandal and T. K. Pal, Int. J. Pharm., 2014, 460, 131-143.

45 V. Bali, M. Ali and J. Ali, Int. J. Pharm., 2011, 403, 46-56.

46 J. Verweij, M. Clavel and B. Chevalier, Ann. Oncol., 1994, 5, 495-505.

47 H. A. Abo Enin, Drug Dev. Ind. Pharm., 2014, 41, 1047-1056.

48 V. Borhade, S. Pathak, S. Sharma and V. Patravale, Int. J. Pharm., 2012, 431, 138-148.

49 S. H. Musa, M. Basri, H. R. Masoumi, R. A. Karjiban, E. A. Malek, H. Basri and A. F. Shamsuddin, Colloids Surf., $B, 2013,112,113-119$.

50 H. O. Ammar, H. A. Salama, M. Ghorab and A. A. Mahmoud, AAPS PharmSciTech, 2009, 10, 808.

51 M. Guttoff, A. H. Saberi and D. J. McClements, Food Chem., 2015, 171, 117-122.

52 F. Ostertag, J. Weiss and D. J. McClements, J. Colloid Interface Sci., 2012, 388, 95-102.

53 C. Qian and D. J. McClements, Food Hydrocolloids, 2011, 25, 1000-1008.

54 A. H. Saberi, Y. Fang and D. J. McClements, J. Colloid Interface Sci., 2013, 391, 95-102.

55 R. Nagarajan, Langmuir, 2002, 18, 31-38.

56 J. Rao and D. J. McClements, J. Food Eng., 2013, 118, 198204. 
57 S. Shafiq, F. Shakeel, S. Talegaonkar, F. J. Ahmad, R. K. Khar and M. Ali, J. Biomed. Nanotechnol., 2007, 3, 28-44.

58 M.-A. Mehrnia, S.-M. Jafari, B. S. Makhmal-Zadeh and Y. Maghsoudlou, Int. J. Biol. Macromol., 2016, 84, 261-267.

59 N. Vankova, S. Tcholakova, N. D. Denkov, I. B. Ivanov, V. D. Vulchev and T. Danner, J. Colloid Interface Sci., 2007, 312, 363.
60 W. Liu, D. Sun, C. Li, Q. Liu and J. Xu, J. Colloid Interface Sci., 2006, 303, 557-563.

61 K. Tong, C. Zhao, Z. Sun and D. Sun, ACS Sustainable Chem. Eng., 2015, 3, 3299-3306.

62 H. Wang and R. H. Davis, J. Colloid Interface Sci., 1993, 159, 108-118. 